Variable Stars as Distance Indicators 


\title{
The Oosterhoff Period Effect and the Age of the Galactic Globular Cluster System
}

\author{
Allan Sandage \\ The Observatories of the Carnegie Institution of Washington \\ 813 Santa Barbara Street, Pasadena, CA 91101, USA
}

\begin{abstract}
The Oosterhoff division of globular clusters into two dichotomous mean period groups is a result of the variation with metallicity of the combined effects of (1) a mean increase in period with decreasing metallicity, and (2) the change of globular cluster horizontal branch (HB) morphology from the M3 to the M13 HB type within the instability strip in the metallicity range of $[\mathrm{Fe} / \mathrm{H}]$ between -1.7 and -1.9 . A new representation of the Oosterhoff period effect showing this property is made from the individual cluster data in Figure 1. The relation between period and metallicity for cluster and for field RR Lyraes at the blue fundamental edge of the instability strip in the HR diagram as read from this figure is

$$
\log P_{a b}=-0.122( \pm 0.02)([\mathrm{Fe} / \mathrm{H}])-0.500( \pm 0.01)
$$

using the metallicity scale of Butler.

The high slope coefficient is consistent with the extant models of the HB when they are read at the varing temperature of the fundamental blue edge given by equation (3) of the text. Most of the current literature treats only the constant temperature condition, which is manifestly incorrect. It is this temperature effect that reconciles the observations and the models.

A new calibration of the absolute magnitudes of RR Lyrae stars as a function of metallicity, combined with new oxygen enhanced isochrones for globular clusters (Bergbusch \& VandenBerg 1992) reduces the age of the Galactic globular cluster system to $14.1 \pm 0.3 \mathrm{Gyr}$ (internal error). The resulting lower age of the universe which, when combined with a Hubble constant near $50 \mathrm{~km}$ $\mathrm{s}^{-1} \mathrm{Mpc}^{-1}$ determined from type I supernovae, shows that the cosmological expansion has been decelerated by an amount consistent with the closure density, permitting $\Omega \sim 1$ now from the timing test.
\end{abstract}

\section{Introduction}

The Oosterhoff $(1939,1944)$ separation of globular clusters into two RR Lyrae period groups and the subsequent discovery that this is also a separation by metallicity (Arp 1955, Kinman 1959) is generally believed to hold a deep clue to the absolute magnitudes of RR Lyrae stars as a function of metallicity. From that, the age of the 
galactic globular clusters follows, leading to the age of the universe, $\mathrm{T}_{0}$, which, by the timing test comparing $T_{0}$ with $\mathrm{H}_{0}$ gives the deceleration parameter, $q_{0}$ from which the eschotology of the cosmos follows.

Recent advances in an understanding of the Oosterhoff effect suggest that such a recalibration of $M(\mathrm{RR})=\mathrm{f}([\mathrm{Fe} / \mathrm{H}])$ can now be made. The method and the result are summarized in this report. The data, precepts, and analysis are set out elsewhere (Sandage 1993, hereafter S93).

In outline, the RR Lyrae luminosity calibration is made through the pulsation equation that relates period, temperature, and mass with luminosity. The first three parameters have been determined as a function of $[\mathrm{Fe} / \mathrm{H}]$ along the fundamental blue edge (hereafter FBE) of the instability strip. The absolute magnitude calibration of the RR Lyrae stars at this edge is then calculated from these parameters.

\section{The Period-Metallicity Relation at the Fundamental Blue Edge}

Figure 1 shows the distribution of observed periods of type RR $a b$ variables in globular clusters of various metallicities. The period data are taken from the Third Catalogue of Variable Stars in Globular Clusters (Sawyer Hogg 1973), updated with more recent data from the literature. The metallicity scale is that of Zinn \& West (1984).

The envelope line defining the shortest period for a given $[\mathrm{Fe} / \mathrm{H}]$ has been determined from the data in Figure 1, corrected to a constant sample size for the different clusters and then combined with similar data for the field RR Lyrae stars in the sample of Blanco (1992). The field star data are based on the metallicity scale of Butler (1975) which is more metal rich by 0.21 dex at a given period than the scale of Zinn \& West.

The equation of the adopted line for the FBE, corrected for sample richness and reduced to the metallicity scale of Butler, is

$$
\log P=-0.122( \pm 0.02)([\mathrm{Fe} / \mathrm{H}])-0.500( \pm 0.01) .
$$

This is the envelope line drawn in Figure 1 near the shortest period for a given metallicity but on the metallicity scale of Zinn \& West.

Figure 1 also shows that the Oosterhoff dichotomy (i.e., into two period groups) is a masquerade of the near discontinuity between $[\mathrm{Fe} / \mathrm{H}]$ of -1.7 and -1.9 . This discontinuity throws the periods for variables that exist in clusters such as M13, M2, M22, NGC 4833, NGC 6809, etc. into the long period group, making an apparent dichotomy. The behaviour is due to the extreme blueness of the HB of many clusters in this metallicity range. Their HB are so blue that they generally miss the instability strip. The variables that do occur have longer periods than they would on the ZAHB because of elevated evolutionary tracks, as was first evident in M13 (Sandage 1970), generalized by Lee, Demarque, and Zinn (1990, hereafter LDZ). This explanation of the Oosterhoff dichotomy was first made by Renzini (1983), Castellani (1983), Lee 
(1990, 1993), and we suspect others.

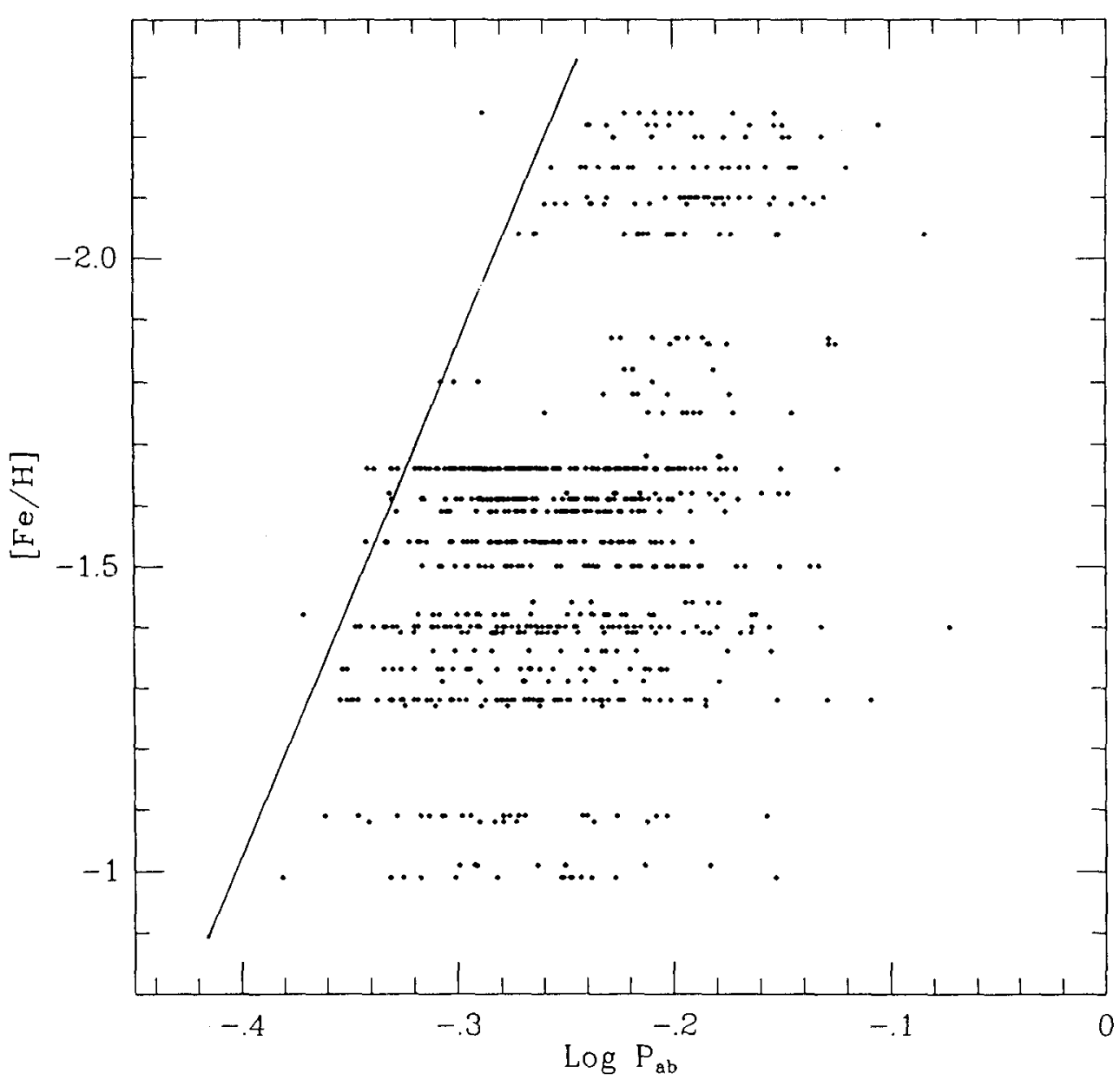

Figure 1. Distribution of observed RR Lyrae periods with metallicity (scale of Zinn \& West) in Galactic globular clusters. The envelope line, reduced to the metallicity scale of Butler (1975), has the slope of equation (1) of the text.

\section{Temperature of the Fundamental Blue Edge as $f([\mathrm{Fe} / \mathrm{H}])$}

Figure 2 shows the variation of the highest temperature at a given metallicity based on the field star sample from Blanco. His new $E(B-V)$ values, applied to $(B-V)_{\text {mag }}$ values from the general catalog of RR Lyrae star parameters by Nikolov, Buchantsova $\&$ Frolov (1984), give unreddened colors. The temperatures are calculated from

$$
\log T_{e}=(0.049[\mathrm{Fe} / \mathrm{H}]-0.288)(B-V)_{\text {mag }}^{0}-0.002[\mathrm{Fe} / \mathrm{H}]+3.931,
$$


based on the atmospheric models of Bell (Sandage 1990, Figure 2) but made cooler by $\Delta \log T_{e}=0.010$ based on models by Kurucz as used by Carney, Storm \& Jones (1992, their equation 13$)$.

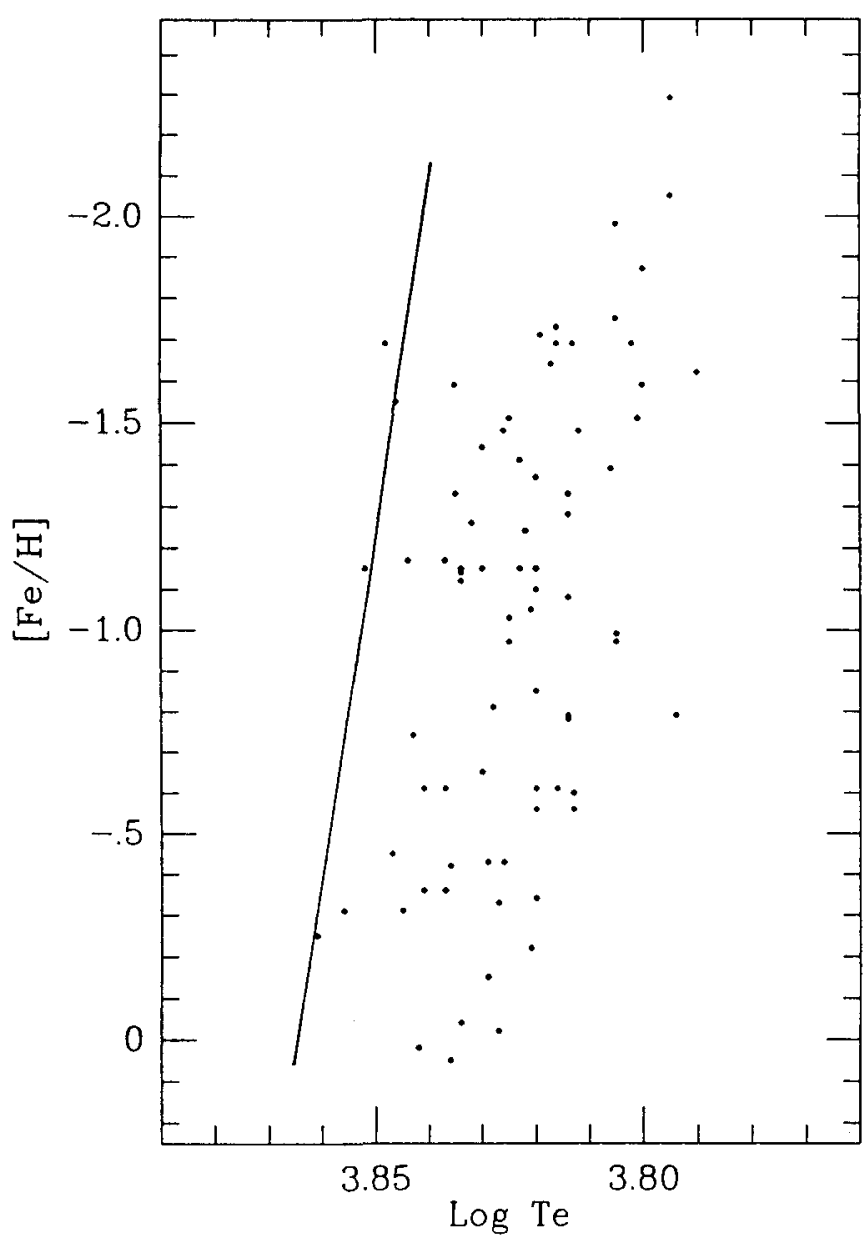

Figure 2. Adopted temperature-metallicity correlation for the fundamental blue edge of the RR Lyrae instability strip. Individual points are from the field star sample of Blanco. The blue envelope is equation (3) of the text.

It has been shown (Preston 1961; Sandage 1990) that the proper color to use for $\mathrm{RR}$ Lyrae stars is $(B-V)_{\mathrm{mag}}$, i.e. integrated over the color curve in magnitudes. It is also shown elsewhere (S93) that the criticism of this type of color by Carney, Storm \& Jones (1992) is not established.

The equation of the line of bluest temperature in Figure 2, taken to define the 
fundamental blue edge, is

$$
\log T_{e}=0.012( \pm 0.01)[\mathrm{Fe} / \mathrm{H}]+3.865( \pm 0.01) .
$$

\section{The Mass-Metallicity Relation at the Fundamental Blue Edge}

It can be shown from the new calculation of masses for double mode RR Lyrae stars by Kovacs et al. (1992, Table 2) using the new Livermore opacities (Iglesias \& Rogers 1991; Rogers \& Iglesias 1992) that the slope of the mass-metallicity relation for the FBE (double mode variables are on the $\mathrm{FBE}$ ) is $d \log \mathcal{M} / d[\mathrm{Fe} / \mathrm{H}]=-0.058$. This also agrees with Dorman's (1992) new oxygen enhanced HB models for the age zero $\mathrm{HB}$ at the blue fundamental edge.

The sense of the correlation of mass with metallicity is the same as for the standard cononical HB models of Sweigart \& Gross (1976) and of Sweigart, Renzini \& Tornambe (1987), but the slope is smaller by about a factor of two. Calculations by Cox (1992), also using the new opacities, give similar results.

The zero point of the mass has been taken from the Dorman models. This zero point has the appropriate property that the masses so determined are consistent with the known distribution of white dwarf masses (but note that these are well known only for population I white dwarfs, Weidemann 1990).

The adopted mass-metallicity relation is

$$
\log \mathcal{M}=-0.058[\mathrm{Fe} / \mathrm{H}]-0.288( \pm 0.01)
$$

From the way we have interpolated in the Dorman tables, equation (4) refers to the fundamental blue edge at the ZAHB.

\section{Exploring the Parameter Space of the Pulsation Equation}

The pulsation equation of van Albada \& Baker (1973) is

$$
\log L=1.19 \log P+0.81 \log \mathcal{M}+4.143 \log T_{e}-13.687 .
$$

with similar equations by Iben (1971) and by Cox (1987). Clearly, in investigations of the slope dependences of $L, \mathcal{M}, P$, and $T_{e}$ on metallicity, the relation $d \log L / d[\mathrm{Fe} / \mathrm{H}]=1.19 d \log P / d[\mathrm{Fe} / \mathrm{H}]+0.81 d \log \mathcal{M} / d[\mathrm{Fe} / \mathrm{H}]+4.143 d \log T_{e} / d[\mathrm{Fe} / \mathrm{H}]$ must hold. Fixing the first term on the right by equation (1) permits an exploration of the permitted variations of the remaining slope coefficients of $L, \mathcal{M}$, and temperature with $[\mathrm{Fe} / \mathrm{H}]$.

Explorations in the relevant parameter space relating $P, L, \mathcal{M}$, and $T_{e}$ using the slope coefficients in equations (1) - (4) shows (S93) that the best value for $d \log L / d[\mathrm{Fe} / \mathrm{H}]$ is $-0.143 \pm 0.05$, or $d M_{\mathrm{bol}} / d[\mathrm{Fe} / \mathrm{H}]=0.36 \pm 0.12$. It is certain that $L(\mathrm{RR})$ must depend on metallicity to explain the Oosterhoff-Arp period variation with metallicity for cluster and for field RR Lyraes (Preston 1959). The introduction of the variation of temperature in the explanation of the Oosterhoff-Arp-Preston 
slope in equation (1) reconciles now the observations and the models. This was first understood for the RRc variables by Simon \& Clement (1993) via a different route.

\section{The RR Lyrae Absolute Magnitude Calibration}

With the slope coefficients and the absolute zero points of the $P, T_{e}$, and $\mathcal{M}$ dependences on metallicity fixed by equations (1)-(4), equation (5) gives

$$
M_{\mathrm{bol}}=0.36( \pm 0.12)[\mathrm{Fe} / \mathrm{H}]+1.00( \pm 0.13) \text {. }
$$

The error on the slope and the error on the zero point are coupled, and only one of the values must be used to assess the overall accuracy of the calibration.

The result in $M_{V}$ is shown in Figure 3 where equation (6) is compared with the summary given elsewhere (Sandage \& Cacciari 1990) of the results of the BaadeWesselink method obtained by (1) Liu \& Janes (1990) whose pioneering analysis of the observations first established beyond doubt that $L$ and $[\mathrm{Fe} / \mathrm{H}]$ are correlated, (2) by Cacciari et al. (1988), (3) by Jones, Carney \& Latham (1988), and by others cited in the aforementioned summary.

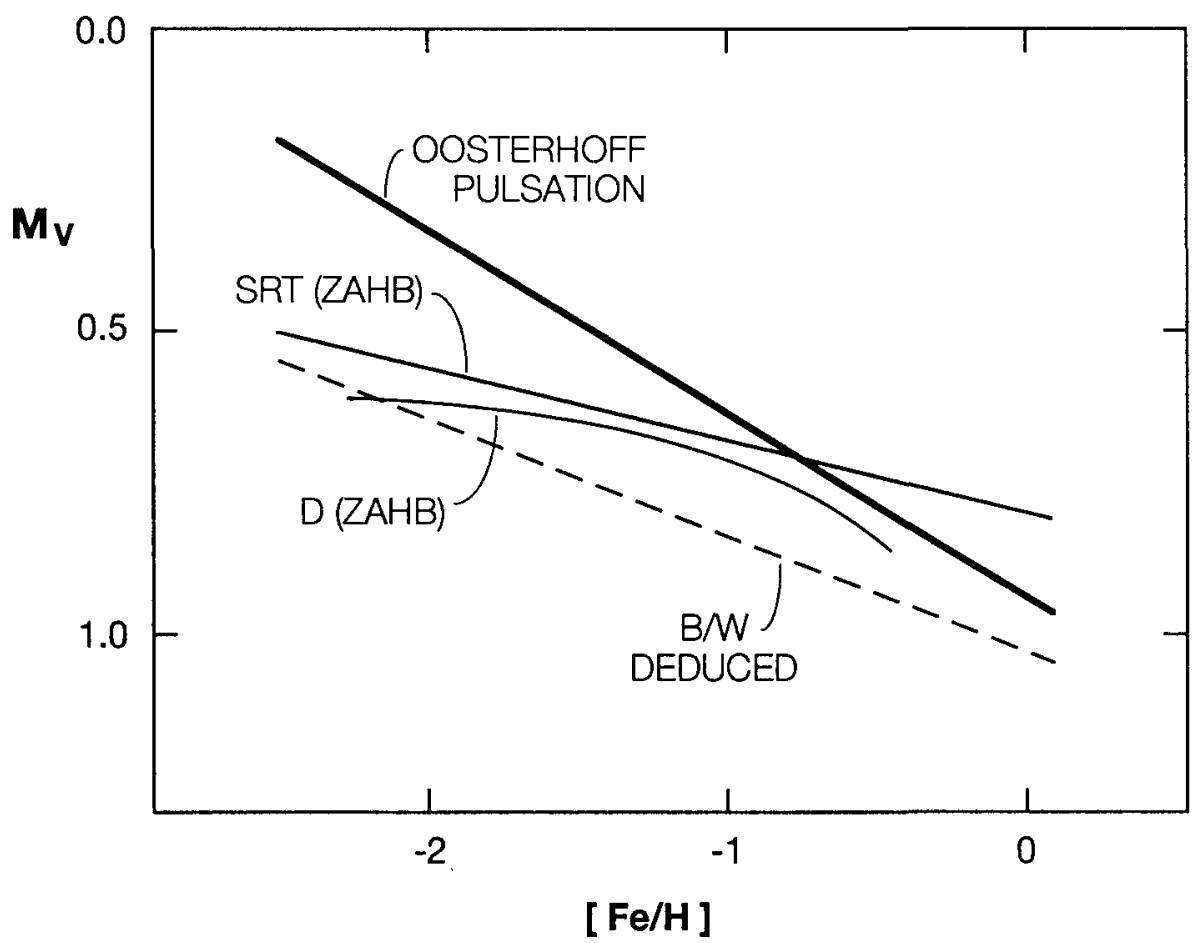

Figure 3. Adopted calibration of the RR Lyraes derived here (labeled "pulsation") compared with other calibrations.

The predictions of the zero age horizontal branch models of Sweigart, Renzini \& 
Tornambe (1987) and of Dorman (1992) are also shown in Figure 3. The predictions from the evolved HB models of Lee, Demarque \& Zinn (1990) are similar to the SRT and $\mathrm{D}$ curves at high metallicities but are brighter by $\sim 0.2$ mag at lower metallicities, agreeing well with equation (6).

Our final calibration is

$$
M_{V}=0.30[\mathrm{Fe} / \mathrm{H}]+0.94
$$

based on the bolometric correction of $\mathrm{BC}=0.06[\mathrm{Fe} / \mathrm{H}]+0.06$, used to convert $M_{\mathrm{bol}}$ to $M_{V}$ for the SRT and D curves in Fig. 3. By the method of derivation, this equation applies to the FBE. Because the HB in $V$ is nearly flat through the instability strip, it should apply closely to most RR Lyrae stars in globular clusters.

Support for this increase in the absolute luminosity of RR Lyrae stars by $\sim 0.2 \mathrm{mag}$ is available from (1) the difference in distance modulus by this amount in galaxies where both classical cepheids and RR Lyrae stars are observed (Saha et al. 1992 for a summary), and (2) the difference in the globular cluster luminosity function between the Galaxy and M31 (Racine \& Harris 1992) based on the older RR Lyrae absolute magnitude calibration (i.e., $\mathrm{B} / \mathrm{W}$ in Figure 3 ). The zero point of the cluster luminosity function in the Galaxy must be made $\sim 0.2$ mag brighter than in M31 using the cepheid distance to M31.

\section{Age of the Galactic Globular Cluster System}

New cluster ages can be calculated (S93) once this RR Lyrae calibration is used to determine the main sequence turnoff luminosity from the observed magnitude difference between the HB and the turnoff. Interpolation in the recent isochrone tables for oxygen enhanced main sequence termination models by Bergbusch \& VandenBerg (1992) gives the age dating equation

$$
\log T(\mathrm{Gyr})=0.39 M_{\mathrm{bol}}(\mathrm{TO})-0.10[\mathrm{Fe} / \mathrm{H}]+8.441 .
$$

The metallicity dependence here is smaller than for the non-oxygen enhanced models used by Sandage \& Caccari (1990, their equations 13-15) based on Ciardullo \& Demarque 1977) and VandenBerg (1983). This is because the oxygen abundance is 250 times higher than for $\mathrm{Fe}$. An increased oxygen abundance increases the effective "metal" abundance.

The ages calculated from equation (8) are shown in Figure 4 for 24 Galactic globular clusters. The mean age is $14.1 \mathrm{Gyr}$, with a formal error of \pm 0.3 . The one sigma range is $\pm 1.4 \mathrm{Gyr}$. The actual range of error for the age of any given cluster is of course larger. Note that an error of only $0.1 \mathrm{mag}$ in the combined steps needed to determine $M_{\text {bol }}(\mathrm{TO})$ gives a $10 \%$ error in the age. Most of the spread in the points in Figure 4 is, then, due to this random uncertainty in the absolute magnitude of the main sequence termination point, although some of the spread has recently been 
documented as real (e.g. Stetson et al. 1989).

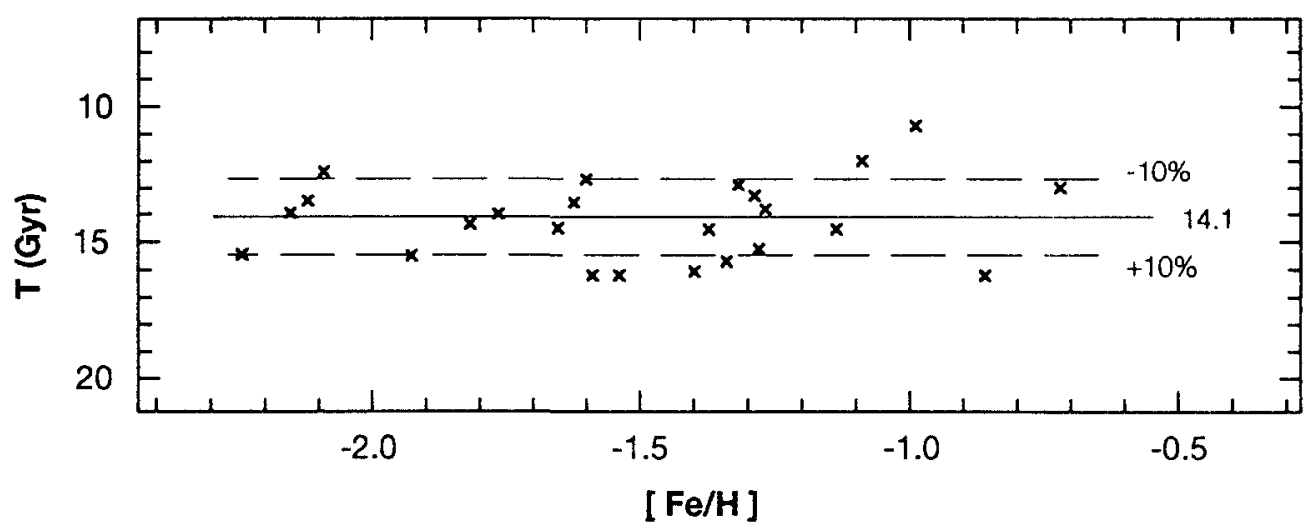

Figure 4. Age of 24 Galactic globular clusters based on the RR Lyrae calibration derived here and on the oxygen enhanced main sequence turnoff models of Bergbusch \& VandenBerg (1992).

Nevertheless, it is incorrect to take the lower envelope of Figure 4 to define the oldest globular clusters in the Galaxy. The statistical error of that envelope is the same as the ridge line. In view of the uncertainty in each of the points by at least $10 \%$, the ridge line gives the correct statistical estimate for the age of the globular cluster system by this method.

\section{The Age of the Universe and the Value of the Cosmological Omega}

The age of the universe is the age of the globular cluster system plus the time for galaxy formation after the initial singularity. No primeval galaxies have yet been found but many theories require the epoch of galaxy formation to be at redshifts of at least $z=10$. The look-back time here is less than $5 \%$ of the present age of the universe.

An observational way to estimate the galaxy gestation time is to note that the look-back time to the quasar with the presently known largest redshift near $z=5$ (Schneider, Schmidt, \& Gunn 1991) is $93 \%$ of the age of the universe in a $q_{0}=1 / 2$ model. (A table of look-back times for various $q_{0}$ values is given elsewhere, Sandage 1961b). If this were to be the time of formation of galaxies, then this would be the same as the age of the globular clusters on the almost certain proposition that globular clusters are the first objects to form in the Galaxy. The age of the universe would then be $14.1+0.96=15$ Gyr with an uncertainty of perhaps \pm 2 Gyr.

Combining such age estimates with the inverse Hubble constant gives the estimate of the deceleration, and hence a direct determination of $\Omega$ shown in Figure 5 . The values of $H_{0}$ here are based on the calibration of the absolute magnitude of the 
prototype supernova 1937C via Cepheids in IC 4182 (Sandage et al. 1993). Each quoted possibility is within the limits posed by the several precepts of the experiment.

The curve gives the $H_{0} T_{0}$ time-scale ratios for various $\Omega$ values $\left(=2 q_{0}\right.$ if $\Omega=$ 0 ) tabulated elsewhere (Sandage 1961a, Table 8). From the ratio of the times as $H_{0} T_{0}=0.66 \pm 0.15$ for the cross and $0.69 \pm 0.17$ for the triangle, calculated from the adopted $T_{0}$ and $H_{0}^{-1}$ values, the formal solution is $\Omega=1(+2,-0.7)$. The ranges are shown as arrows along the abscissa.

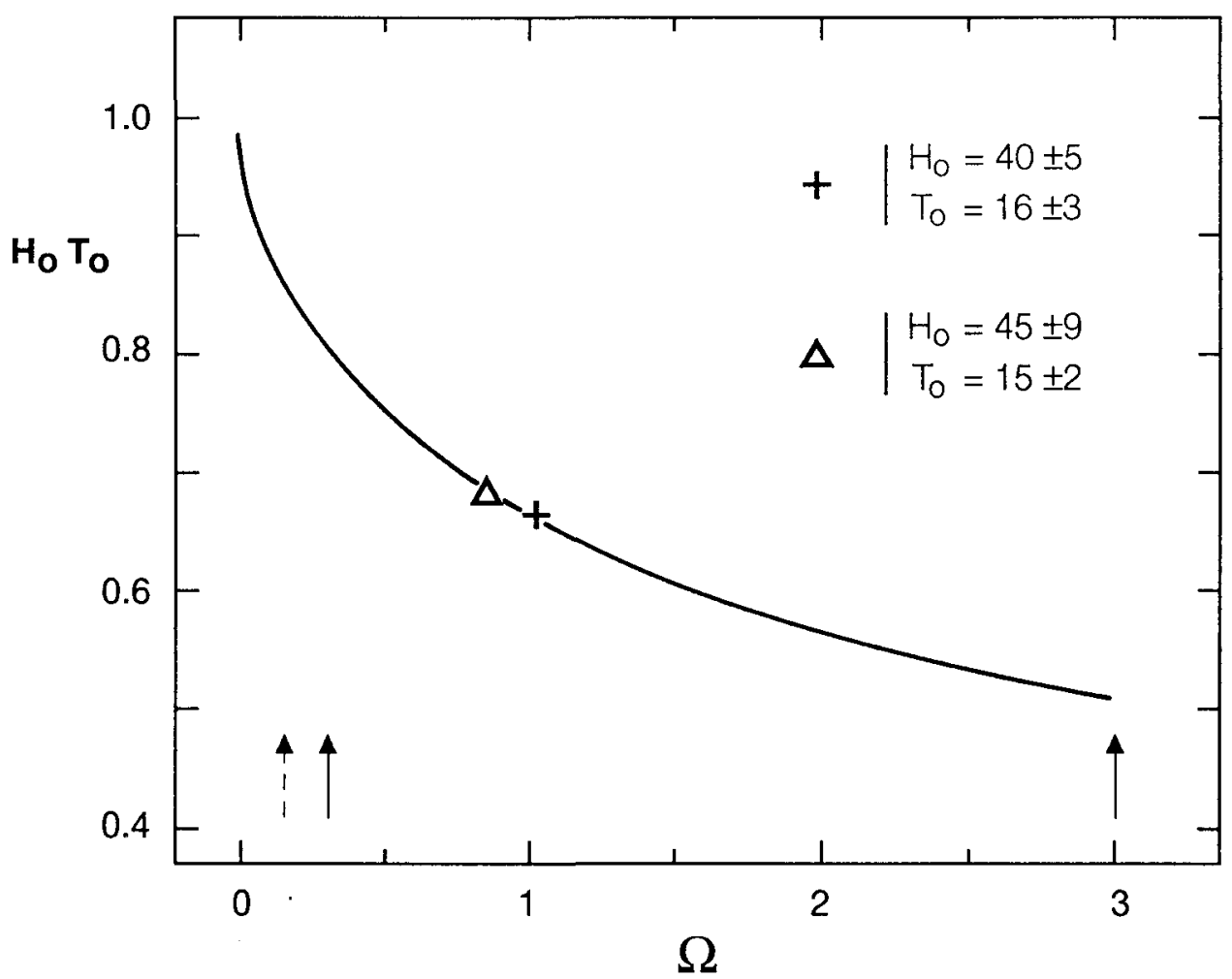

Figure 5. The ratio of the age of the universe to the the inverse Hubble constant as a function of $\Omega$. The two inferred "observed" points are shown as cross and triangle.

The conclusion is that the new calibration of the absolute magnitude of RR Lyrae stars at $\sim 0.2 \mathrm{mag}$ brighter than previous calibrations at $[\mathrm{Fe} / \mathrm{H}] \sim-1.8$, together with the new oxygen enhanced main-sequence-termination isochrones, reduce the ages of the Galactic globular clusters to $14 \mathrm{Gyr}$. The new value of $H_{0}$ via type I supernovae gives a Hubble time near $24 \mathrm{Gyr}$. If true, there has been a deceleration of the expansion sufficient to accomodate a closure density, giving $\Omega \sim 1$ directly from 
the timing argument.

It is a pleasure to acknowledge the hospitality of G. A. Tammann and the astronomers at the Astronomisches Institut der Universität Basel where this report was written during an extended visit in the summer of 1992 after the Victoria meeting. Thanks are also extended to K. Cardy in Pasadena and D. Cerrito in Basel for producing the diagrams, and to James Nemec for assistance in preparing the camera-ready manuscript.

\section{References:}

Arp, H. C. 1955, AJ, 60, 317

Bergbusch, P. A., \& VandenBerg, D. A. 1992, ApJS, 81, 163

Blanco, V. 1992, AJ, 104, 734

Butler, D. 1975, ApJ, 200, 68

Castellani, V. 1983, Mem. Soc. Astr. Ital., 54, 141

Carney, B. W., Storm, J., \& Jones, R. V. 1992, ApJ, 386, 663

Ciardullo, R. B., \& Demarque, P. 1977, Trans. Yale Obs., Vol. 33

Cacciari, C., Clementini, G., Prevot, L., \& Buser, R. 1988, A\&A, 209, 141

Cox, A. N. 1987 in IAU Colloq. 95, Second Conference on Faint Blue Stars, ed. A.G.D. Philip, D.S. Hayes, \& J.W. Liebert (Schenectady: Davis), p 161 1992, private communication

Dorman, B. 1992, ApJS, 81, 221

Iben, 1. 1971, PASP, 83, 697

Iglesias, C. A. \& Rogers, F. J. 1991, ApJL, 371, 73

Jones, R. V., Carney, B. W., \& Latham, D. W. 1988, ApJ, 332, 206

Kinman, T. D. 1959, MNRAS, 119, 538

Kovacs, G., Buchler, J. R., Marom, A., Iglesias, C. A., \& Rogers, F. J., 1992, A\&A, 259, L46

Lee, Y.-W. 1990, PhD thesis, Yale Univ. 1993, these proceedings, pp. 285-294.

Lee, Y.-W., Demarque, P., \& Zinn, R. 1990, ApJ, 350, 155

Liu, T., \& Janes, K. A. 1990, ApJ, 354, 273

Nikolov, N., Buchantsova, N. \& Frolov, M. 1984, Catalog of RR Lyrae Photometric Properties; Astron. Council, USSR Acad Sci., Sofia.

Oosterhoff, P. Th. 1939, Observatory, 62, 104

_ 1944, Bull. Astr. Inst. Netherlands, 10, 55

Preston. G. W. 1959, ApJ, 130, 507

—— 1961, ApJ, 133, 29

Racine, R., \& Harris, W. E. 1992, AJ, 104, 1068

Renzini, A. 1983, Mem. Soc. Astr. Ital., 54, 335

Rogers, F. J., \& Iglesias, C. A. 1992, ApJS, 79, 507

Saha, A., Freedman, W. L., Hoessel, J. G., \& Mossman, A. E. 1992, AJ., 104, 1072

Sandage, A. 1961a, ApJ, 333, 355

- 1961b, ApJ, 334, 916 
- 1990, ApJ, 350, 603

- 1993, AJ, submitted (S93)

Sandage, A. \& Cacciari, C. 1990, ApJ, 350, 645

Sandage, A., Saha, A., Tammann, G. A., Panagia, N., \& Macchetto, D. 1993, ApJL, in press

Sawyer Hogg, H. 1939, David Dunlop Obs. Pub., 1, No. 4

- 1955 , ibid, 2 , No. 4

- 1973, ibid, 3 , No. 6

Schneider, D. P., Schmidt, M., \& Gunn, J. E. 1991, AJ, 102, 837

Simon, N. R. \& Clement, C. M. 1993, these proceedings, pp. 304-312.

Stetson, P., VandenBerg, D. A., Bolte, M., Hesser, J. E., \& Smith, G. H. 1989, AJ, 97, 1360

Sweigart, A. V., \& Gross, P. G., 1976, ApJS, 32, 367

Sweigart, A., Renzini, A., \& Tornambe, A, 1987, ApJ, 312, 762

van Albada, T. S., \& Baker, N. 1973, ApJ, 185, 477

VandenBerg, D. A. 1983, ApJS, 51, 29

Weidemann, V. 1990, ARA\&A, 28, 103

Zinn, R. \& West, M. J. 1984, ApJS, 55, 45

\section{DISCUSSION}

C.CACCIARI: RR Lyrae masses of about 0.8 solar masses, as recently found using the Lawrence-Livermore opacities, are significantly larger than the values found by stellar evolution for HB stars, and are a problem for the interpretation of HB morphologies. Could you please comment on that?

A.SANDAGE: You refer to the fact that the mass at the tip of the RGB is also $\sim 0.8$ solar masses, leaving no room for mass loss to populate the HB along its length? Due to the apparently high sensitivity of the mass to $[\mathrm{Fe} / \mathrm{H}] \mathrm{I}$ could believe that the exact values of calculated $\mathrm{HB}$ masses are uncertain, but that the trend in $[\mathrm{Fe} / \mathrm{H}]$ might be believed - but $I$ agree it is a real problem if the calculated mass of 0.8 solar masses is set in stone. The Dorman model masses used in the final version of his paper are lower than I had in the verbal presentation, and should be fine for this problem.

Y.-W. LEE: The crucial point of the Oosterhoff dichotomy is why two clusters of more or less the same $[\mathrm{Fe} / \mathrm{H}]$ (e.g., M2 and M3) have such a large difference in $\left\langle P_{a b}\right\rangle$, and hence produce the gap in $\left\langle P_{a b}\right\rangle$. That this can be explained by the blueward shift of the $\mathrm{HB}$ morphology with decreasing $[\mathrm{Fe} / \mathrm{H}]$ and accompanying evolutionary effect has been announced by me two years ago in Bologna (see also Lee in this volume). Renzini and Castellani noted that the non-monotonic behavior of the HB morphology with decreasing $[\mathrm{Fe} / \mathrm{H}]$ has something to do with the Oosterhoff effect, but they did 
not mention specifically the importance of the redward evolution in the way explained by Lee and LDZ. Hence they do not deserve the credit on that point, and also they did not explain the origin of the Oosterhoff gap.

A. SANDAGE: Thank you. It is clear that your evolutionary ideas for M13 type, extreme blue $\mathrm{HB}$ clusters have such high $\log \left\langle P_{a b}\right\rangle$ values even in the range $[\mathrm{Fe} / \mathrm{H}]$ between -1.5 and -1.8 . This is the prime reason for the dichotomy into the 0.65 OoII bin and why that bin is so sharp. But there is no LDZ evolution in the OoI bin where both red and blue parts of the HB are on the ZAHB. The density distribution through the instability strip must also be taken into account in any detailed discussion of why the $\log \left\langle P_{a b}\right\rangle=\mathrm{f}([\mathrm{Fe} / \mathrm{H}])$ plot shows two nearly vertical distributions (at $\left\langle P_{a b}\right\rangle$ near 0.55 and 0.65 day) rather than the continuous $\log P_{a b}=\mathrm{f}([\mathrm{Fe} / \mathrm{H}])$ shown by the blue fundamental edge plots both for the cluster data and for the field RR Lyrae stars (Blanco's new $E(B-V)$ and $\Delta S$ data).

B.DORMAN: (question concerning masses of metal-poor HB stars)

A. SANDAGE: For $[\mathrm{Fe} / \mathrm{H}]=-2.26$ one obtains 0.80 solar masses at $15 \mathrm{Gyr}$, and 0.84 at 12 Gyr. Masses of 0.80 solar masses are thus possible, but only if there is little mass loss. The inference of no mass loss is at least "uncomfortable". However, for example, the M15 HB morphology seems to exclude the scaled solar models which give this mass in the instability strip. In this case you must have masses of between 0.70 and 0.75 solar masses. So there is still a "mass problem" if Cox's mass value continues to stand. Note that the above-quoted masses are completely independent of $\mathrm{CNO}$ abundance.

Of course there must be mass loss from the top of the AGB to the ZAHB to populate the $\mathrm{HB}$ along its length. I believe one cannot believe the absolute values of $\operatorname{Mass}(\mathrm{RR})$ to within say $\Delta \log \mathcal{M} \sim 0.1$, from theoretical HB models (various calculations based on different opacities [Kovacs, Cox, Livermore vs. Los Alamos, etc] $]$, but one can believe (I think) required trends of mass $=\mathrm{f}([\mathrm{Fe} / \mathrm{H}])$. 